perception in refractory GORD. Increased air swallowing (aerophagia) is often suspected based on clinical evaluation. More recently, increased air swallowing between meals was demonstrated, using oesophageal impedance, in a group of patients with increased abdominal gas (x-ray). Aerophagia during meals, however, may be more relevant for GORD patients with postprandial symptoms. We hypothesised that mealtime air swallowing may impact on postprandial reflux patterns and symptoms in patients with refractory GORD. We aimed to assess aerophagia during meals and postprandial gas reflux in GORD patients, responding or refractory to PPI. Methods Mealtime air swallows were quantified using ambulatory impedance-pH monitoring. Normal values were established from 39 healthy controls (mean age 39, range 22-62; Shay et al 2004). We studied 44 consecutive patients (mean age 48, range 19-78) with typical reflux symptoms and pathological oesophageal $24 \mathrm{~h}$ acid exposure. 18 were fully responsive and 26 were partially or unresponsive to PPI. Mealtime air swallows were defined as swallows with fast impedance increase ( $>3000 \Omega$ from baseline) in the distal recording segment. Mealtime air swallow frequency (air swallows/ 10 min meal) was calculated.

Results There was no difference in mealtime air swallow frequency (mean \pm SEM $8.6 \pm 1.0$ vs $8.0 \pm 0.7$ per $10 \mathrm{~min}$ ) or total mealtime air swallows (67.1 \pm 8.3 vs $54.0 \pm 5.3)$ between GORD patients and controls. In the GORD group, PPI-refractory patients had a higher frequency $(10.5 \pm 1.4$ vs $5.9 \pm 0.8, \mathrm{p}<0.05)$ and number $(83.1 \pm 12.7 \mathrm{vs}$ $47.8 \pm 7.3, p<0.05)$ of mealtime air swallows compared to PPIresponders. PPI-refractory patients had a higher number $(25.5 \pm 4.0$ vs $16.8 \pm 3.3, \mathrm{p}<0.05)$ and proportion $(70.3 \pm 3.0 \%$ vs $54.0 \pm 6.0 \%$, $\mathrm{p}<0.05$ ) of post-prandial gas-containing reflux episodes than PPIresponders. There was no difference between GORD patients in fasting air swallowing or $24 \mathrm{~h}$ acid exposure.

Conclusion GORD patients had similar mealtime air swallowing to controls, but both groups had large inter-individual variability. Within GORD patients, PPI non-responders had more mealtime air swallowing than responders. Consequently non-responders had more reflux episodes containing gas, an important factor in reflux perception in GORD patients, who have hypersensitivity to oesophageal distension. Mealtime air swallowing may be amenable to behavioural therapy as an "add on" treatment in patients with incomplete response to PPI and objective aerophagia during meals.

Competing interests None declared.

\section{OC-094 ASSESSMENT OF OBSTRUCTIVE DEFECATION BY HIGH RESOLUTION MANOMETRY COMPARED TO MAGNETIC RESONANCE DEFECOGRAPHY}

doi:10.1136/gutjnl-2012-302514a.94

${ }^{1} \mathrm{H}$ Heinrich,* ${ }^{1} \mathrm{H}$ Fruehauf, ${ }^{1} \mathrm{M}$ Fried, ${ }^{1} \mathrm{M}$ Sauter, ${ }^{1,2} \mathrm{M}$ R Fox. ${ }^{1}$ Division of Gastroenterology, University Hospital Zurich, Zurich, Switzerland; ${ }^{2}$ NIHR Biomedical Research Unit, Nottingham Digestive Diseases Centre, Nottingham, UK

Introduction Patients with obstructive defecation may have abnormalities of anorectal function or structure. Standard investigation by manometry shows only fair agreement with patient symptoms and defecography. Anorectal high resolution manometry (HRM) may improve diagnostic accuracy by differentiating pressure effects caused by contraction vs straining and by avoiding movement artifacts.

Aims This study compared HRM findings with magnetic resonance (MR) defecography in the clinical assessment of patients with obstructive defecation defined by Rome III criteria as straining at stool with the sensation of incomplete evacuation, blockage sensation or digital facilitation.

Methods HRM was performed by a solid state catheter with 10 circumferential sensors at $6 \mathrm{~mm}$ separation across the anal canal and two placed $5 \mathrm{~cm}$ proximal in the rectum (Manoscan AR 360, Given) Resting tone, squeeze pressure and dynamic pressure activity during bearing down were analysed. Findings were referred to MR defecography (1.5T, Philips) performed with $250 \mathrm{ml}$ enema labelled with paramagnetic contrast as reference standard for pelvic floor anatomy and function.

Results 188 consecutive patients (155 female; age 19-93) referred with symptoms of obstructive defecation had full investigation. MRI diagnosis revealed anorectal dyssynergia with paradoxical contraction in 59 and structural pathology in 124 patients (rectocele $(n=68)$ and rectocele with intususception $(n=33)$, pelvic floor descent with enterocele $(n=28)$ or prolapse $(n=7)$, pelvic floor descent $(n=87)$ most patients had multiple pathologies. Five patients were excluded because of inconclusive MRI findings. Compared to patients with dyssynergia, those with structural pathology had lower resting (65 vs $86 \mathrm{~mm} \mathrm{Hg}$; $<0.003$ ); and squeeze pressure (150 vs $181 \mathrm{~mm} \mathrm{Hg;} \mathrm{c}$ 0.011). On simulated defecation a negative rectoanal pressure gradient was more evident in dyssynergia than structural pathology $(p<0.0001)$. In patients with dyssynergia on MRI, HRM showed paradoxical contraction or failure to increase abdominal pressure without anal relaxation (sensitivity 100\% (59/59); specificity 94\% (117/124)). One patient with normal findings on MRI but paradoxical contraction on HRM had an anal fissure. A pattern of high intra-rectal pressure with a steep, positive pressure gradient indicating outlet obstruction in the anal canal was observed on HRM in $n=26$ patients with rectocele with intussusception on MRI.

Conclusion Diagnostic agreement between anorectal HRM and MR defecography is high and pressure measurements accurately identify recto-anal dyssynergia. A steep intraanal pressure gradient is indicative of outlet obstruction by structural pathology as a cause of obstructive defecation.

Competing interests None declared.

\section{Gastroduodenal free papers OC-095 ASSESSMENT OF CANDIDATE GENES TO ASSIST PROGNOSIS IN GASTRIC CANCER}

doi:10.1136/gutjnl-2012-302514a.95

A Gelsthorpe,* M McFarlane, S James, R Arasaradnam, H Mehanna, C Nwokolo. Department of Gastroenterology, University Hospital of Coventry, Coventry, UK

Introduction Gastric cancer remains the fourth most prevalent cancer and the second leading cause of cancer-related death in the world. Prognostic models for survival are inadequate. In this retrospective study of archival material we used a panel of 32 RNA probes to characterise gene expression in gastric cancer patients with aggressive disease and those who survived long term.

Methods The University Hospital pathology database was searched for all gastric adenocarcinomas diagnosed between April 2005 and September 2006. The patients' age, sex, tumour stage and survival was recorded. Sixty cancers $(n=60)$ were identified; paraffin sections retrieved and processed using HTG Molecular's qNPA technology to hybridise RNA probes specific to each gene sequence. Tissues sections were placed directly into the 96 plate wells before hybridisation and automated reading. Each plate contained generic anchor sequences hybridised to linker probes, creating gene-specific hybridisation spots to measure each qNPA probe. Each tissue section was analysed 2 or 3 times, gene expression quantitated and an average numerical value derived. Data sets were analysed for normal distribution using the Kolmogorov-Smirnov statistic method. T-test was used with Welch correction on those samples which passed while Mann-Whitney test was used on those that did not. The expression of the 32 genes was compared between patients with metastatic disease $(n=30)$ and 5 -year survivors $(n=10)$. 
Results When patients with metastatic disease were compared with 5 -year survivors significant increased gene expression was noted for heat shock protein (HSP-90), $p=0.016$ and KRAS, $p=0.046$. When the pattern of gene expression between those who survived less than a year $(n=40)$ and those surviving 5 years $(n=10)$ was compared then HSP-90 and GHRL were shown also to have altered expression. A variety of genes including TFT1, HSPD1, BCAS1, CAPDH, GHRL were globally enhanced among virtually all samples. Conclusion HSP-90 a gene encoding a chaperone protein implicated in carcinogenesis exhibited increased expression in metastatic disease. Up-regulation of HSP-90 should help cancer cells adapt to stress conferring a survival advantage. Thus finding relative overexpression in cancers which have progressed to metastatic disease suggests a possible role as a prognostic marker. This study has identified candidate genes that could contribute to a prognostic model for gastric cancer utilising qNPA technology and demonstrates the up-regulation of HSP-90 and KRAS in advanced gastric cancer.

Competing interests None declared.

\section{OC-096 TH17 CELLS ARE INCREASED IN HELICOBACTER PYLORI INFECTION AND MAY BE ASSOCIATED WITH PEPTIC ULCER DISEASE}

doi:10.1136/gutjnl-2012-302514a.96

E Staples, R J M Ingram, ${ }^{*} \mathrm{~K}$ Hussain, D P Letley, J C Atherton, K Robinson. Univesity of Nottingham, Nottingham, UK

Introduction Helicobacter pylori $(\mathrm{Hp})$ persistently colonises the stomachs of half the world's population. The majority of hosts remain asymptomatic but 10\%-15\% will develop peptic ulcer disease (PUD) or gastric cancer. Disease is associated with an IFN $\gamma$ producing T-helper 1 (Th1) response. Pro-inflammatory IL-17producing T-helper 17 (Th17) cells, which express the transcription factor RORC2, are also likely to be involved. The murine Th17 response to $H_{p}$ has been characterised but the role of human Th17 responses remains unclear.

Aim To assess the importance of Th17 cells in $\mathrm{Hp}+$ patients. We quantified Th17 cells in the human gastric mucosa, compared IL17, RORC2 and IFNG transcription, and investigated correlations with PUD.

Methods Gastric biopsies were donated by patients undergoing routine upper GI endoscopy at Queen's Medical Centre in Nottingham with informed consent and ethical approval. Patient characteristics: 17 with $\mathrm{Hp}$-associated PUD, 28 with $\mathrm{Hp}$-associated gastritis and 17 uninfected ( $H p-)$. Antral IL17 and RORC2 mRNA levels (all patient biopsies) and IFNG transcription (26 Hp + and 9 $\mathrm{Hp}$ - biopsies) and were quantified by real time PCR (RT-qPCR) relative to a comparator prepared from a further 14 uninfected biopsies. Frequencies of IL-17-secreting $\mathrm{CD}^{+}$and $\mathrm{CD}^{+}{ }^{+} \mathrm{T}$-cells were assessed by flow cytometry in gastric biopsies donated by 12 $\mathrm{Hp}+$ and $11 \mathrm{Hp}$ - patients.

Analysis Comparisons between $H p+$ and $H p$ - groups and patients with and without PUD used Mann-Whitney tests. Levels of mRNA expression for paired biopsies were compared with Wilcoxon signed rank tests. Spearman's rank correlation was used to analyse relationships.

Results Increased frequencies of $\mathrm{CD}^{+} \mathrm{IL}-17^{+}$Th17 (3.0-fold, $\mathrm{p}=0.001$ ) and $\mathrm{CD}^{+} \mathrm{IL}-17^{+}$(Tc17) cells (3.3-fold, $\mathrm{p}=0.01$ ) were present in $H p+$ samples. RT-qPCR showed that infected patients have increased mucosal IL17 (45.0-fold, $\mathrm{p}<0.0001)$ and IFNG expression (3.4-fold, $\mathrm{p}=0.006$ ) and showed for the first time that $R O R C 2$ expression was also higher (2.6-fold, $\mathrm{p}<0.0001)$. There was a trend towards a correlation between IL17 and IFNG expression $(\mathrm{r}=0.39, \mathrm{p}=0.051)$ and relative IL17 expression was 3.1-fold higher than IFNG ( $\mathrm{p}=0.0006)$. Relative RORC2 gene expression was also $35 \%$ higher in tissue from $\mathrm{Hp}+$ patients with PUD than in those with gastritis alone but this did not reach statistical significance $(\mathrm{p}=0.11)$.

\section{Conclusion}

1. The Hp-infected human stomach has increased frequencies of Th17 and Tc17 cells, and increased expression of IL17, RORC2 and IFNG.

2. IL17 expression was significantly higher than IFNG expression, though the sources of IL17 have yet to be fully characterised. There was a trend for increased RORC2 expression in PUD. Our data suggest that Th17 cell responses may influence the clinical outcome of $H_{p}$ infection in patients.

Competing interests None declared.

\section{OC-097 THE NOVEL GASTRIN/CCK2 RECEPTOR ANTAGONIST YF476 INDUCES CLINICAL RESPONSES AND IS WELL TOLERATED IN PATIENTS WITH TYPE I GASTRIC NEUROENDOCRINE TUMOURS}

doi:10.1136/gutjnl-2012-302514a.97

${ }^{1}$ A R Moore, ${ }^{*} \mathrm{~L}$ Ball, ${ }^{2} \mathrm{M}$ Boyce, ${ }^{1} \mathrm{~A}$ Varro, ${ }^{1} \mathrm{D}$ M Pritchard. ${ }^{1}$ Institute of Translational Medicine, University of Liverpool, Liverpool, UK; ${ }^{2}$ Hammersmith Medicines Research, London, UK

Introduction Autoimmune chronic atrophic gastritis causes loss of gastric parietal cells and results in achlorhydria, increased antral gastrin production and hypergastrinaemia. In some patients this hypergastrinaemia induces hyperplasia of enterochromaffin-like (ECL) cells and leads to type I gastric neuroendocrine (carcinoid) tumour (NET) development. Most type I gastric NETs behave in an indolent fashion, but a small proportion $(<1 \%)$ grow more rapidly and metastasise. Surgical antrectomy has been shown to lead to resolution of hypergastrinaemia and regression of tumours in some patients. We therefore hypothesised that pharmacological inhibition of the gastrin/CCK-2 receptor using the novel orally bioavailable competitive antagonist YF476 would also lead to clinical regression of type I gastric NETs. The aims of this study were to assess (1) whether YF476 is an effective medical treatment for type I gastric neuroendocrine tumours; (2) the safety and tolerability of YF476 treatment and (3) the effects of YF476 on biomarkers of ECL cell activity.

Methods Following ethical committee and MHRA approval, six patients with small type I NETs secondary to autoimmune chronic atrophic gastritis and hypergastrinaemia have received a 12-week course of $50 \mathrm{mg}$ /day YF476. Clinical responses were monitored by six weekly upper GI endoscopy with biopsy and three weekly measurement of fasting serum gastrin and chromogranin A (CgA) concentrations. Drug tolerability has been assessed by monitoring clinical adverse events and by assessing haematological, renal and hepatic blood parameters.

Results In all six patients the number and size of NETs decreased following 12 weeks of therapy (mean reduction in size of largest tumour $=39 \%$, mean reduction in tumour number $=40 \%$ ). However no patient showed complete tumour regression after 12 weeks' therapy. Serum CgA concentrations decreased in all subjects while receiving YF476, but increased to pre-treatment levels in the three subjects in whom measurements have to date been performed 12 weeks after completing therapy. Fasting serum gastrin concentrations did not significantly change while patients received YF476. YF476 was well tolerated in all patients; no serious adverse effects were reported and there was no evidence of haematological, renal or hepatic toxicity. 